\title{
MMP-2 and TIMP-1 predict healing of WTC-lung injury in New York City firefighters
}

\author{
Anna Nolan ${ }^{1,2,3 \dagger}$, Sophia Kwon ${ }^{1 \dagger}$, Soo Jung Cho ${ }^{1}$, Bushra Naveed ${ }^{1,3}$, Ashley L Comfort ${ }^{1}$, David J Prezant ${ }^{4 \dagger}$,
} William N Rom ${ }^{1,2}$ and Michael D Weiden ${ }^{1,2,3,5^{*}}$

\begin{abstract}
Rationale: After 9/11/2001, most FDNY workers had persistent lung function decline but some exposed workers recovered. We hypothesized that the protease/anti-protease balance in serum soon after exposure predicts subsequent recovery.

Methods: We performed a nested case-control study measuring biomarkers in serum drawn before 3/2002 and subsequent forced expiratory volume at one second $\left(\mathrm{FEV}_{1}\right)$ on repeat spirometry before $3 / 2008$. Serum was assayed for matrix metalloproteinases (MMP-1,2,3,7,8,9,12 and 13) and tissue inhibitors of metalloproteinases (TIMP-1,2,3,4). The representative sub-cohort defined analyte distribution and a concentration above $75^{\text {th }}$ percentile defined elevated biomarker expression. An $\mathrm{FEV}_{1}$ one standard deviation above the mean defined resistance to airway injury. Logistic regression was adjusted for pre-9/11 FEV $1, \mathrm{BMI}$, age and exposure intensity modeled the association between elevated biomarker expression and above average $\mathrm{FEV}_{1}$.
\end{abstract}

Results: $\mathrm{FEV}_{1}$ in cases and controls declined 10\% of after 9/11/2001. Cases subsequently returned to $99 \%$ of their pre-exposure FEV while decline persisted in controls. Elevated TIMP-1 and MMP-2 increased the odds of resistance by 5.4 and 4.2 fold while elevated MMP-1 decreased it by 0.27 fold.

Conclusions: Resistant cases displayed healing, returning to 99\% of pre-exposure values. High TIMP-1 and MMP-2 predict healing. MMP/TIMP balance reflects independent pathways to airway injury and repair after WTC exposure.

Keywords: Biomarkers, Lung disease, Occupational exposure

\section{Introduction}

The collapse of the World Trade Center (WTC) on 9/11/ 2001 produced a massive exposure to dust and products of combustion [1-3]. The Fire Department of New York (FDNY) bureau of health services rapidly responded to this atrocity, initiating a medical monitoring program in October of 2001. Over 13,000 exposed rescue workers have been longitudinally followed by the FDNY-WTCMedical Monitoring and Treatment Program. Approximately 7,000 exposed workers had serum samples drawn, stored and $\mathrm{FEV}_{1}$ and FVC measured within six months of $9 / 11 / 2001$. A vast majority of those exposed had an acute

\footnotetext{
* Correspondence: michael.weiden@med.nyu.edu

${ }^{\dagger}$ Equal contributors

'Division of Pulmonary, Critical Care and Sleep, New York University, School of Medicine, 462 First Avenue, NB7N24, New York, NY, USA

${ }^{2}$ Department of Environmental Medicine, New York University, School of

Medicine, 57 Old Forge Road, Tuxedo Park, NY, USA

Full list of author information is available at the end of the article
}

decline in lung function in the first six months followed by stabilization. There was no recovery in lung function for the group as a whole, but a minority of those exposed recovered lung function over the following six and a half years.

We used serum obtained soon after the exposure to measure serum biomarkers of lung injury during the process of disease evolution. We reported that inflammatory cytokines, lipids and other measure of metabolic syndrome as well as biomarkers of cardiovascular risk predict abnormal lung function years later [4-6].

The balance of increased protease activity and reduced anti-protease activity are components of many diseases including cigarette-induced chronic lung disease and other causes of accelerated lung function decline [7-10]. Genetic association studies with matrix metalloproteinases (MMPs) demonstrate a strong association with the development of lung disease [7]. MMP-1 is induced in smokers with COPD and its overexpression in mice causes emphysema [11,12].

\section{Biomed Central}


The destructive effects of MMPs are inhibited by tissue inhibitors of matrix metalloproteinases (TIMPs). Since most clinical investigation focuses on disease, there is little data on the role of MMPs and TIMPs in the resistance to the damaging effect of dust exposure [13-16]. One carefully done pathologic study demonstrated increased MMP-2 and TIMP-1 mRNA expression in surgically removed lung and predicted improved $\mathrm{FEV}_{1}$ in COPD patients [17]. Serum MMP and TIMP expression reflects the severity of COPD supporting an investigation of a link between serum MMP/TIMP balance and lung function in the WTC exposed cohort [18].

This study investigates serum expression of MMPs and TIMPs soon after damaging particulate matter exposure and tests if protease/anti-protease balance is associated with a subgroup that demonstrates an above average healing potential after WTC-LI. We report that elevated TIMP-1 and MMP-2 predicts recovery of lung function while elevated MMP-1 reduces the odds of recovery years after WTC exposure.

\section{Methods}

\section{Study participants and design}

The Institutional Review Boards (IRB) of NYU and Montefiore Medical Center approved this study and patients signed consent at the time of serum draw within 6 months of 9/11/2001 (Montefiore Medical Center IRB; \#07-09-320 and New York University IRB; \#11-00439). The parent cohort for this investigation consists of 1,720 exposed workers who required subspecialty pulmonary examination (SPE) prior to 3/10/2008.

A nested case-control study tested the association of serum biomarkers and $\mathrm{FEV}_{1}$ at SPE. The baseline cohort $\mathrm{N}=801$ was assembled to exclude patients with abnormal pre-9/11 $\mathrm{FEV}_{1}$ and tobacco use to eliminate these confounders of post exposure lung function [19]. Resistant cases $(\mathrm{N}=100 / 801)$ had a $\mathrm{FEV}_{1} \%$ one standard deviation above the mean $(>107 \%)$ at SPE. Controls $(\mathrm{N}=171 / 801)$ were randomly selected after stratification of the baseline cohort for BMI and $\mathrm{FEV}_{1}$. Serum was available for $\mathrm{N}=$ $137 / 171$ of the cohort controls, $N=77 / 100$ of the resistant cases. For this case-control study, the control subjects are all individuals in the random sample cohort control who did not meet criteria to be resistant cases. Analyte distribution in the cohort controls identified the $75^{\text {th }}$ percentile cut points used to define elevated biomarker expression.

\section{Serum biomarker assays}

Processing of blood has been previously described $[6,20]$. Biomarkers were assayed with a TIMP panel (R\&D Systems, Minneapolis, MN), and MMP panel (Procarta/Affymetrix) using a Luminex 200-IS (Luminex Corporation, Austin, TX). Each plate contained 1:2 ratio of resistant cases to controls to account for batch effect.

\section{Chest computed tomography (CT)}

We have reported on lung function and computed tomography $(\mathrm{CT})$ findings of this group. High-resolution chest CT scans were obtained with 7-mm thick mages reconstructed at 6-mm intervals. Images were read for airway and parenchymal abnormalities by dedicated, board certified radiologists who had no knowledge of the subjects' exposure status or clinical findings. Bronchial wall thickening and air-trapping were interpreted qualitatively and analyzed as a yes/no variable.

\section{Statistical analysis}

Database management and statistics used SPSS 20 (IBM, Armonk, NY). Odd ratios were modeled with multivariate binary logistic regression with case status as the outcome. Analyte cutoff values were the $75^{\text {th }}$ percentile cohort control expression. The Hosmer-Lemeshow goodness-of-fit test was used to assess calibration of the final model. The model discrimination was evaluated using the receiver operating characteristic area under the curve (AUC).

\section{Results \\ Participants}

Derivation of cases and controls is depicted in Figure 1. N $=111 / 171$ cohort controls with serum available for study were compared to $\mathrm{N}=77 / 100$ resistant cases. Controls were not significantly different from the baseline cohort in arrival time, years of service, age, time to all pulmonary examinations, or BMI, Table 1. Controls had higher BMI than resistant cases. Time to medical monitoring entry (MME), when spirometry was performed and serum drawn, was 2 months in cases and controls. Time to subspecialty pulmonary evaluation SPE, when spirometry that defined case status was performed, was 32 months in cases and controls.

Longitudinal lung function in resistant cases and controls Cases and controls underwent three longitudinal measures of lung function. The first spirometry documented pre-exposure lung function; the second was immediately after exposure at MME and the third was later at SPE, Table 2 and Figure 2. Resistant cases had higher median pre-9/11 $\mathrm{FEV}_{1}$ than controls $(117 \%$ vs $98 \% \mathrm{p}<0.001)$.

$\mathrm{FEV}_{1}$ declined in cases and controls soon after exposure. To test if group data represented individuals' response the ratio of $\mathrm{FEV}_{1}$ pre-9/11 to $\mathrm{FEV}_{1}$ at MME was calculated for each case and controls. The mean (SD) of MME/Pre-911 ratio was $0.91(0.10)$ for resistant cases and 0.91 (0.12) for controls ( $\mathrm{p}=0.95$ for cases vs control). Cases recovered most of the lost $\mathrm{FEV}_{1}$ (117\% to $\left.113 \%\right)$. Controls had less improvement than cases in their median $\mathrm{FEV}_{1}$ (98\% to 93\%). To test if group data represented individuals' response the ratio of $\mathrm{FEV}_{1}$ pre-9/11 to $\mathrm{FEV}_{1}$ at SPE was calculated for each case and controls. The SPE/Pre-911 ratio 


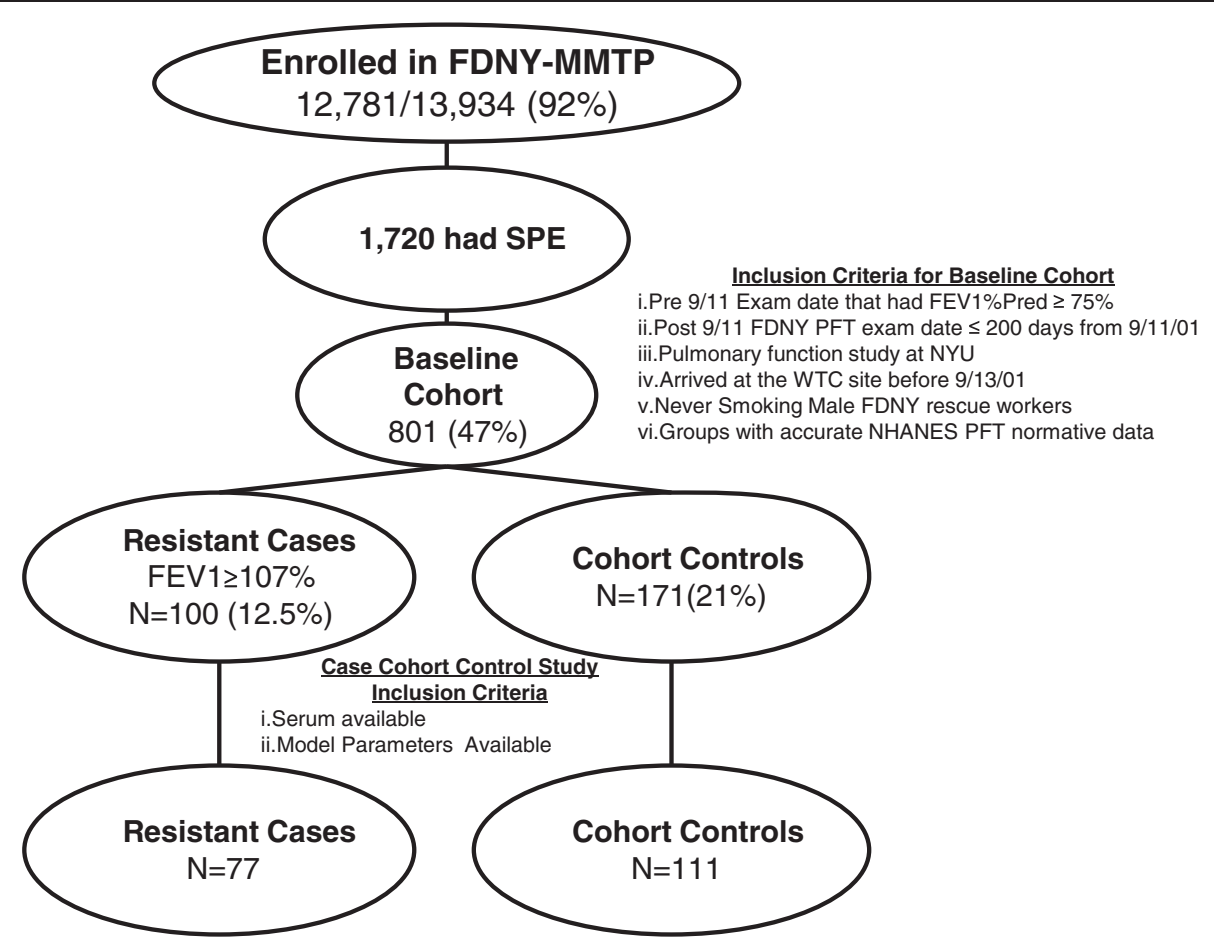

Figure 1 Study design. Derivation of cases $n=77$ and controls $n=111$ from the FDNY-MMTP.

was $0.99+/-0.09$ for resistant cases and $0.91+/-0.12$ for controls ( $\mathrm{p}<0.001$ for cases vs control). Raw $\mathrm{FEV}_{1} \%$ predicted has also been shown in Figure 2.

Both groups had a decline in $\mathrm{FEV}_{1} / \mathrm{FVC}$ ratio SPE/MME, with resistant cases having the less than controls (0.85 to $0.81 \mathrm{p}<0.001)$. Both cases and controls had a high proportion of individuals with bronchodilator response (22\% vs $28 \%$ NS). Cases had less methacholine reactivity when measured as a continuous slope ( 0.64 vs $0.04 \mathrm{p}<0.01)$; $9 \%$ of cases reactive to a $10 \mathrm{mg} / \mathrm{ml}$ dose while $20 \%$ of controls were reactive $(p=0.11)$. Cases had less air trapping than controls $(111 \%$ vs $121 \% \mathrm{p}<0.02)$ when residual volume was used to measure air trapping Table 3. At SPE, resistant cases had the higher TLC, DLCO and alveolar volume than controls ( $\mathrm{p}<0.001$ for all comparisons), Table 2 .

\section{Chest CT of resistant cases and controls}

Chest imaging was used to investigate if resistant cases and controls had structural differences. In those with chest CT images available, $14 \%$ of the resistant cases had bronchial wall thickening whereas $35 \%$ of the controls had this evidence of airway inflammation $(\mathrm{p}<0.03)$, Table 3 . There was no significant difference between cases and controls in air trapping defined by mosaic attenuation (28\% vs $46 \%$ $\mathrm{p}=0.09)$. Both cases and controls have a high proportion of pulmonary nodules ( $38 \%$ vs $36 \% \mathrm{p}=0.8$ ).

\section{Biomarker models}

Analyte levels were compared between controls and resistant cases, Table 4 . We used logistic regression with analyte expression above a pre-defined $75^{\text {th }}$ percentile cut points

Table 1 Demographics

\begin{tabular}{|c|c|c|c|c|c|}
\hline & Date/event & Baseline cohort & Sub-cohort controls & Resistant cases & $p$ \\
\hline \multirow[t]{2}{*}{ WTC Exposure, n(\%) } & High & $197(25)$ & $21(19)$ & $14(18)$ & 1.000 \\
\hline & Intermediate & $604(75)$ & $90(81)$ & $63(82)$ & \\
\hline \multirow[t]{2}{*}{ 9/11 to PFT, months } & MME & $2.7(2.0-3.8)$ & $2.5(2.0-3.2)$ & $2.6(2.2-3.2)$ & 0.219 \\
\hline & SPE & $33.8(24.8-57.0)$ & $32.7(24.6-57.4)$ & $32.5(28.3-52.2)$ & 0.755 \\
\hline \multirow[t]{2}{*}{$\mathrm{BMl}, \mathrm{kg} / \mathrm{m} 2$} & MME & $28.0(26-30)$ & $28.1(26-31)$ & $27.3(26-29)$ & 0.018 \\
\hline & SPE & $28.9(27-31)$ & $29.1(27-31)$ & $27.6(26-30)$ & 0.010 \\
\hline Years of service & 9/11/01 & $13(7-19)$ & $14(7-18)$ & $14(10-19)$ & 0.249 \\
\hline Age & 9/11/01 & $40(36-45)$ & $41(36-44)$ & $42(38-46)$ & 0.159 \\
\hline
\end{tabular}

WTC World trade center, PFT Pulmonary function test, BMI Body mass index, MME Medical monitoring entry, SPE Subspecialty pulmonary exam. Expressed as median (interquartile range. 
Table 2 Longitudinal lung function assessment of cohort

\begin{tabular}{|c|c|c|c|c|}
\hline Time & Variable & Controls & Resistant & $p$ \\
\hline \multirow[t]{2}{*}{ Pre-9/11 } & FEV1\% & $98(90-108)$ & $117(108-124)$ & $<0.0001$ \\
\hline & FEV1/FVC & $84(81-87)$ & $87(84-89)$ & $<0.0001$ \\
\hline \multirow[t]{2}{*}{ MME } & FEV1\% & $91(81-96)$ & 105(97-113) & $<0.0001$ \\
\hline & FEV1/FVC & $84(79-87)$ & 85 (83-89) & $<0.0001$ \\
\hline \multirow[t]{11}{*}{ SPE } & FEV1\% & $93(83-98)$ & $113(109-119)$ & $<0.0001$ \\
\hline & FEV1/FVC & $77(73-79)$ & $81(79-84)$ & $<0.0001$ \\
\hline & $\mathrm{BD}$ response & $5(2-10)$ & $5(2-9)$ & 0.776 \\
\hline & $\mathrm{BD}$ response $>12 \%, \mathrm{~N}(\%)$ & $13 / 46(28)$ & $5 / 23(22)$ & 0.772 \\
\hline & MCT slope & $0.64(0.31-0.15)$ & $0.04(0.02-0.07)$ & 0.010 \\
\hline & PC20 < 10 mg/mL MCT, N (\%) & 17/86 (20) & $6 / 66(9)$ & 0.108 \\
\hline & TLC & $103(95-109)$ & $106(103-116)$ & 0.016 \\
\hline & $\mathrm{RV}, \%$ Pred & $125(113-141)$ & $111(103-123)$ & 0.015 \\
\hline & DLCO & $106(99-114)$ & $116(108-131)$ & 0.001 \\
\hline & VA & $93(85-98)$ & $100(95-105)$ & $<0.0001$ \\
\hline & DLCONA & $120(107-130)$ & $110(107-126)$ & 0.358 \\
\hline
\end{tabular}

FEV1 Forced expiratory volume in one second, FVC Forced vital capacity, MCT Methacholine, BD Bronchodilator, $P C 20$ concentration of methacholine causing a $20 \%$ fall in FEV1, DLCO Diffusion capacity of the lung for carbon monoxide, VA Alveolar volume.

to test if protease or anti-proteases expression predicted resistance to WTC-LI [21]. Models were adjusted for BMI, age, exposure group and pre-9/11 $\mathrm{FEV}_{1}$. Reduced models examined the ability of a single analyte to predict each case definition. Analytes with significant odds ratios in single biomarker models were used to develop the final multi-analyte model.

Using $75^{\text {th }}$ percentile of cohort expression, we calculated OR with FEV1 $>107 \%$ at SPE as the outcome. Elevated MMP-1 reduces the odds of FEV1 $>107 \%$ by $31 \%$, elevated MMP-2 increases the odds of FEV1 > $107 \%$ by $218 \%$ and elevated TIMP-1 increases the odds by $231 \%$. After adjusting for BMI, age, exposure group and pre-9/11 FEV $\mathrm{Fl}_{1}$ elevated

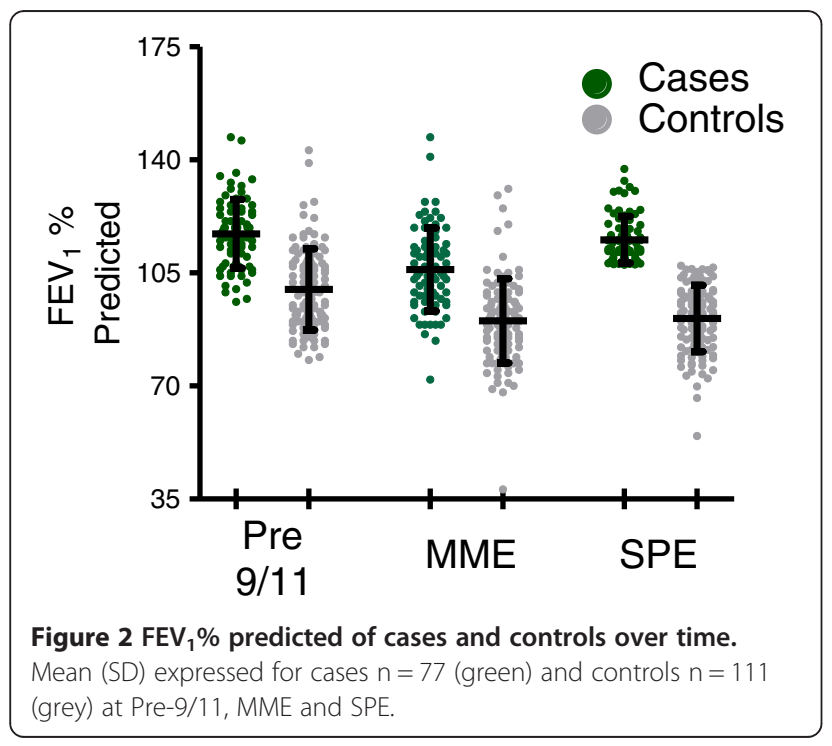

MMP-1 reduces the odds of resistance to WTC-LI by $68 \%$ while individuals with high MMP-2 and TIMP-1 were $300 \%$ and 350\% more likely to resist WTC-LI, Table 4. Combining MMP-1, MMP-2 and TIMP-1 in a multianalyte model improved the OR for each of these biomarkers, Table 5. Elevated MMP-1 was a risk factor reducing the odds of resistance to lung injury to 0.27 (95\% CI 0.09-0.82 $\mathrm{p}=0.02)$. Elevated MMP-2 and TIMP-1 were protective factors improving odds of resistance by 4.2 fold (95\% CI 1.6-10.8 $\mathrm{p}=0.003)$ and 5.4 fold (95\% CI 1.9-14.9 $\mathrm{p}=0.001)$, Table 5 . The area under the receiver operator curve for the multi-analyte model was 0.90 (95\% CI; 0.860.94). (Figure 3) The sensitivity and specificity of the model was $74 \%$ and $86 \%$ respectively.

\section{Discussion}

We report that elevated MMP-1, MMP-2 and TIMP-1 in serum shortly after WTC exposure predicts subsequent return of $\mathrm{FEV}_{1}$ to pre-exposure values. All subjects in this nested case control investigation had significant WTC dust exposure, arriving at the collapse site within 2 days of $9 / 11 / 2001$. The FDNY measured serial $\mathrm{FEV}_{1}$ pre and post $9 / 11$. We focused our current study on a subgroup of highly exposed individuals who did not suffer persistent

Table 3 Chest CT abnormalities in cases and controls

\begin{tabular}{llll}
\hline CT Finding & Controls & Resistant cases & pt \\
\hline Bronchial wall thickening & $23 / 65(35)$ & $5 / 36(14)$ & 0.022 \\
Air trapping & $30 / 65(46)$ & $10 / 36(28)$ & 0.090 \\
Nodules & $25 / 65(38)$ & $13 / 36(36)$ & 0.834 \\
\hline
\end{tabular}

${ }^{\dagger}$ Significance calculated by Chi-Squared. 
Table 4 Serum biomarkers

\begin{tabular}{|c|c|c|c|c|c|c|c|}
\hline & \multirow{2}{*}{$\begin{array}{l}\text { Analyte } \\
\mathrm{pg} / \mathrm{mL}\end{array}$} & \multirow{2}{*}{$\begin{array}{c}\text { 75th } \\
\text { percentile cutpiont }\end{array}$} & \multicolumn{2}{|l|}{ Controls } & \multicolumn{2}{|l|}{ Resistant } & \multirow[t]{2}{*}{ OR $(95 \% \mathrm{CI})$} \\
\hline & & & Median (IQR) & Proportion & Median (IQR) & Proportion & \\
\hline \multirow[t]{8}{*}{ Proteases } & MMP-1 & 1239 & $689(264-1210)$ & $27 / 111$ & $549(233-1013)$ & $14 / 77$ & $0.691(0.335-1.425)$ \\
\hline & MMP-2 & 4949 & $2933(1570.01-4608.25)$ & $22 / 111$ & 3340 (2108.19-5682.13) & $27 / 77$ & $2.185(1.128-4.231)$ \\
\hline & MMP-3 & 13345 & 7494 (2940-13358) & 28/111 & 6906 (3457-11509) & $17 / 77$ & $0.840(0.422-1.671)$ \\
\hline & MMP-7 & 355 & $217(100-333)$ & $25 / 111$ & $237(95-375)$ & $22 / 77$ & $1.376(0.707-2 .-77)$ \\
\hline & MMP-8 & 157 & $10(10-156)$ & $27 / 111$ & $10(10-116)$ & $16 / 77$ & $0.816(0.405-1.645)$ \\
\hline & MMP-9 & 62086 & 22559 (10596-40806) & $24 / 111$ & 23003 (12043-64398) & $21 / 77$ & $1.359(0.692-2.670)$ \\
\hline & MMP-12 & 306 & $66(18-306)$ & 28/111 & $55(27-277)$ & $19 / 77$ & $0.971(0.496-1.902)$ \\
\hline & MMP-13 & 138 & $75(10-133)$ & $26 / 111$ & $71(10-140)$ & $20 / 77$ & $1.147(0.585-2.248)$ \\
\hline \multirow[t]{4}{*}{ Anti-proteases } & TIMP-1 & 155003 & 125491 (100396-151151) & $23 / 111$ & $126292(100483-164661)$ & $29 / 77$ & $2.312(1.206-4.430)$ \\
\hline & TIMP-2 & 115905 & 102470 (89901-113738) & $23 / 111$ & 103199 (88959-119820) & $22 / 77$ & $1.530(0.780-3.005)$ \\
\hline & TIMP-3 & 31922 & 7750 (7750-31922) & $29 / 111$ & 7750 (7750-33680) & $21 / 77$ & $1.060(0.550-2.044)$ \\
\hline & TIMP-4 & 1585 & $1242(9288-1562)$ & $26 / 111$ & 1342 (970-(1717) & $27 / 77$ & $1.765(0.929-3.354)$ \\
\hline
\end{tabular}

MMP matrix metalloproteinase, TIMP Tissue inhibitor of matrix metalloproteinase, OR Odds RATIO (unadjusted), IQR Interquartile range.

$\mathrm{FEV}_{1}$ decline. This resistant subgroup had greater than average reduction in $\mathrm{FEV}_{1}$ immediately after exposure but returned to pre-exposure $\mathrm{FEV}_{1}$ over the next 6.5 years. Because serum was drawn well before the pulmonary function test that demonstrated recovery, the biomarker information reflected the evolving response to injury. MMP-2 and TIMP-1 expression above the $75^{\text {th }}$ percentile are protective biomarkers, significantly increasing the odds of resistance between 4.2 and 5.4 fold. Alternately, elevated MMP-1 is a risk factor, reducing the odds of resistance by $73 \%$. The biomarker model using serum MMP-1, MMP-2 and TIMP-1 concentration predicted resistance with a sensitivity of $74 \%$, a specificity of $86 \%$ and a receiver operator characteristic of 0.90 .

As expected in this highly exposed group, both cases and controls suffered an acute reduction in $\mathrm{FEV}_{1}$ as a result of WTC exposure. Resistant cases differed from controls because they returned to $99 \%$ of their pre-exposure $\mathrm{FEV}_{1}$. Over the 6.5 years post $9 / 11 \mathrm{FEV}_{1}$ returned to only $91 \%$ of their pre-exposure $\mathrm{FEV}_{1}$. The return of $\mathrm{FEV}_{1}$ to preexposure levels provides evidence that resistant cases have above average capacity to heal after an acute injury.

Table 5 Model of resistance to WTC-lung injury

\begin{tabular}{llc}
\hline \multicolumn{1}{c}{$\begin{array}{c}\text { Serum biomarker } \\
\text { 75th percentile cutpoint }\end{array}$} & $\begin{array}{c}\text { OR (95\% Cl) } \\
\text { Adjusted§ }\end{array}$ \\
\hline Single analyte & MMP-1 $\geq 1239$ & $0.33(0.11-0.93)$ \\
& MMP-2 $\geq 4949$ & $3.00(1.25-7.18)$ \\
Multi-analyte & TIMP-1 $\geq 155003$ & $3.52(1.41-8.81)$ \\
& MMP-1 $\geq 1239$ & $0.27(0.09-0.82)$ \\
& MMP-2 $\geq 4949$ & $4.16(1.61-10.76)$ \\
& TIMP-1 $\geq 155003$ & $5.38(1.94-14.94)$ \\
\hline
\end{tabular}

§Models Adjusted for: BMI at SPE, exposure Group, Age at 9/11 and Pre-9/11 FEV 1 \% Predicted; -2 Log Likelihood 144.081; Hosmer and Lemeshow (Goodness of Fit) Sig 0.743 .
Elevated TIMP-1 and MMP-2 expression increases the odds of being resistant 4.1 and 5.3 fold respectively. These results remain significant after adjusting for multiple comparisons. Although TIMP and MMP over expression have been observed in COPD and are probably affected by chronic injury secondary to cigarette smoke or other damaging processes, interpreting the cause and effect relationship between lung function and MMP/TIMP balance in humans has been challenging [22-24]. A carefully done study of 63 patients who had surgery for lung cancer or lung transplantation demonstrated that increasing TIMP-1 and MMP-2 mRNA in the small airways and/or

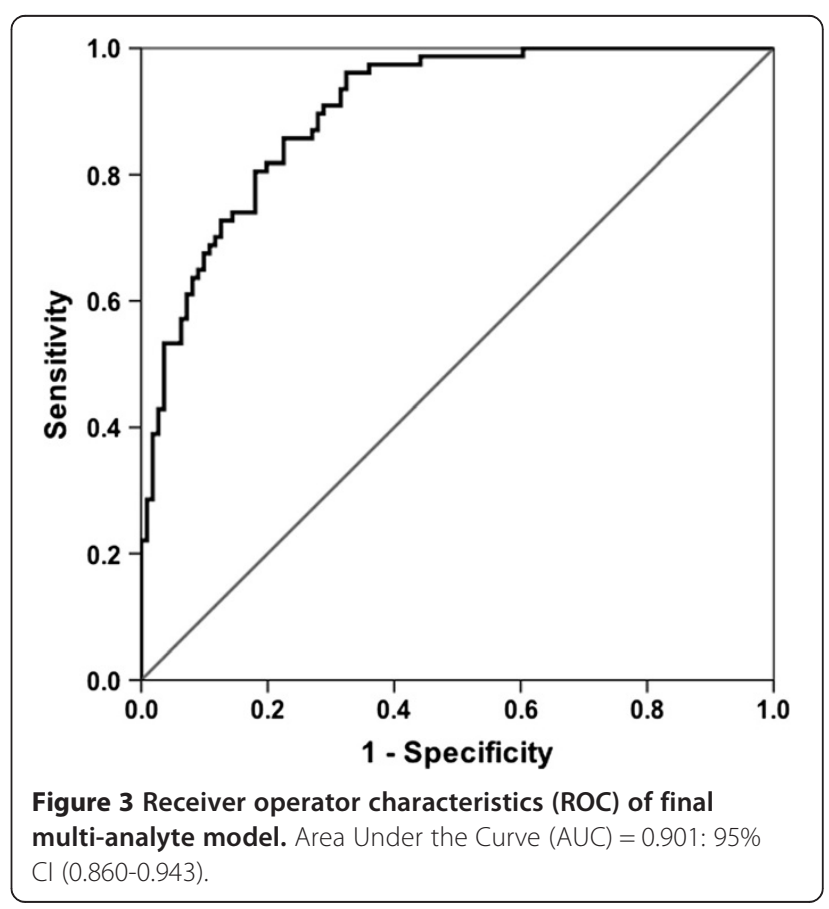


the parenchyma surrounding the small airway was positively associated with $\mathrm{FEV}_{1}$ [17]. Our findings on particulate matter induced lung injury are consistent with these findings in smoking related COPD patients. The mechanisms involved in the protective effects of TIMP1 and MMP-2 in response to particulate matter induced lung injury require further study.

Since resistant cases were defined by a $\mathrm{FEV}_{1}$ of $>107 \%$, starting close to this threshold should increase the likelihood of crossing this defining value. After adjusting the logistic model for pre-9/11 FEV1, the association of MMP-1 MMP-2 and TIMP-1 with better than average post exposure $\mathrm{FEV}_{1}$ markedly improved as quantified by their ORs. This suggests that while pre-existing differences in lung function is a confounder including them in the regression allowed the biomarker-lung function association to remain robust. We briefly examined if excluding individuals who failed their MCT by the $3^{\text {rd }}$ level would alter our results. We found that the ORs of MMP-1, MMP-2 and TIMP-1 remained significant and of the same magnitude (data not shown). Furthermore, we repeated the analysis in a population with their Pre-9/11 $\mathrm{FEV}_{1} \%$ predicted constrained; cases $\mathrm{n}=58$ had an $\mathrm{FEV}_{1}$ of 96-146 and controls $\mathrm{n}=58$ had an $\mathrm{FEV}_{1}$ of $96-143$. Using this constrained population OR (95\% CI) were as follows: MMP-1 0.318 (0.098-1.039), MMP-2 4.993 (1.664-14.983) and TIMP-1 8.061 (2.423-26.825). Importantly, patients with resistance to WTC-LI had at subspecialty pulmonary evaluation increased TLC measured on plethysmography, increased alveolar volume measured by methane dilution and increased diffusion of carbon monoxide than controls. In animal models, MMP-2 is required for normal lung development and failure to produce MMP-2 leads to emphysema and collagen deposition around the bronchioles [25-27]. Our data is consistent with the hypothesis that MMP-2 and TIMP-1 are biomarkers of an individual's intrinsic capacity to heal after irritant induced lung injury.

The resistant cases also had significantly less bronchial wall thickening on chest CT. Accumulation of inflammatory cells in the bronchovascular bundle likely accounts for this radiographic finding [28]. Our Chest CT findings suggest that the resistant group accumulates fewer inflammatory cells around the bronchovascular bundle after dust exposure. Interestingly, in rodent models of lung injury, MMP-2 expression reduces bronchovascular inflammatory cells and enhances repair $[29,30]$. Since the chest CT was performed years after the insult, the mechanism that produced the bronchial wall thickening persists for years after the original exposure that precipitated the inflammation.

Conversely, elevated MMP-1 reduced the odds of being resistant to WTC-LI to 0.27 . This association, however, becomes insignificant when adjusted for multiple comparisons. We have maintained MMP-1 in the model because of the overwhelming evidence that this protease is an important is disease pathogenesis. Over expression of MMP-1 leads to emphysema in animal models [31]. In humans, MMP-1 is expressed in type II pneumocytes of patients with COPD but not controls $[11,12]$. The $73 \%$ reduction in the odds of having above average $\mathrm{FEV}_{1}$ is consistent with the damaging effects of this protease on lung integrity.

Since we excluded ever smokers and individuals with pre-9/11 lung disease, our results are not confounded by these common causes of $\mathrm{FEV}_{1}$ decline that are unrelated to inhalation of WTC dust. Another advantage of this study is the SPE PFT was performed during the initial pulmonary evaluation prior to treatment initiation. Therefore our results are not confounded by treatment effect. In spite of our objective case definition, using $\mathrm{FEV}_{1}$ as a single measure did produce misclassification of disease. The resistant group with $\mathrm{FEV}_{1}>107 \%$ at SPE did have a individuals with evidence of airway injury. Up to $22 \%$ of the resistant cases had airway reactivity on PFT or radiographic evidence of bronchial wall thickening. This misclassification of disease should bias toward the null. In spite of this bias, we observed highly significant associations between biomarkers in serum drawn soon after exposure and above average lung function years later.

This nested case control study has several limitations. The cohort was highly unusual, suffering an acute overwhelming exposure to PM that overwhelmed normal protective mechanisms. The results therefore have limited generalizability. The findings require replication in independent particulate matter exposed cohorts. Even though the serum biomarkers were expressed years before the $\mathrm{FEV}_{1}$ that defined resistance to lung injury, the results are correlations and do not imply causation. It is possible that exposure lead to an alteration in these biomarkers due to different mechanisms controlling lung injury. Alternately, pre-existing attenuation of these biomarkers may lead to differential healing. Further, investigation in model systems and longitudinally followed cohorts is required to better understand the role, if any, of MMP-1 and TIMP-1 in healing after PM induced lung injury. Finally, this study had no unexposed control group because the few unexposed workers were markedly different from the exposed group with poor health that prevented them from working at the WTC collapse site. This restricts our ability to assess the impact of WTC exposure to the observed biomarker disease relationship.

This report documents the serum biomarkers that predict better than average $\mathrm{FEV}_{1}$ after massive dust exposure. This group had evidence of healing with return to pre-9/11 $\mathrm{FEV}_{1}$ after a significant drop immediately post exposure. The processes initiated by WTC exposure impacted multiple distinct injury and repair pathways. One interpretation of the findings is that biomarkers of resistance reflect biological processes leading to healing after particulate matter induced injury. 


\section{Competing interests}

The authors report no financial or competing interests.

\section{Authors' contributions}

AN, SK and MDW participated in study conception and design. AN, SK and MDW were the primary investigators. AN, SK, ALC, BN and MDW were responsible for data collection. AN, SJC and SK were responsible for data validation. AN, ALC and SK participated in data analysis. AN, SK undertook the statistical analysis. All authors participated in data interpretation, writing and revision of the report and approval of the final version. All authors read and approved the final manuscript

\section{Funding sources}

T32ES007267, K23HL084191/S1, K24A1080298, R01HL057879, R01A1067522 and UL1 TR000038.

\section{Author details}

${ }^{1}$ Division of Pulmonary, Critical Care and Sleep, New York University, School of Medicine, 462 First Avenue, NB7N24, New York, NY, USA. ${ }^{2}$ Department of Environmental Medicine, New York University, School of Medicine, 57 Old Forge Road, Tuxedo Park, NY, USA. ${ }^{3}$ Fire Department of New York, Bureau of Health Services and Office of Medical Affairs, Brooklyn, NY, USA. ${ }^{4}$ Department of Medicine, Pulmonary Medicine Division, Montefiore Medical Center and Albert Einstein College of Medicine, Bronx, NY, USA. ${ }^{5}$ Department of Medicine and Environmental Medicine, 550 1st Avenue, New York, NY 10016, USA.

Received: 12 April 2013 Accepted: 10 December 2013 Published: 21 January 2014

\section{References}

1. Landrigan PJ: Health consequences of the 11 September 2001 attacks. Environ Health Perspect 2001, 109(11):A514-A515. Epub 2001/11/20.

2. Claudio L: Environmental aftermath. Environ Health Perspect 2001, 109(11): A528-A536. Epub 2001/11/20.

3. Aldrich TK, Gustave J, Hall CB, Cohen HW, Webber MP, Zeig-Owens R, et al: Lung function in rescue workers at the World Trade Center after 7 years. N Engl J Med 2010, 362(14):1263-72. Epub 2010/04/09.

4. Weiden MD, Naveed B, Kwon S, Segal LN, Cho SJ, Tsukiji J, et al: Comparison of WTC dust size on macrophage inflammatory cytokine release in vivo and in vitro. PLoS One 2012, 7(7):e40016. Epub 2012/07/21.

5. Naveed B, Weiden MD, Kwon S, Gracely EJ, Comfort AL, Ferrier N, et al: Metabolic syndrome biomarkers predict lung function impairment: a nested case-control study. Am J Respir Crit Care Med 2012, 185(4):392-399. Epub 2011/11/19.

6. Nolan A, Naveed B, Comfort AL, Ferrier N, Hall CB, Kwon S, et al: Inflammatory biomarkers predict airflow obstruction after exposure to World Trade Center dust. Chest 2011, 142(2):412-8. Epub 2011/10/15.

7. Hunninghake GM, Cho MH, Tesfaigzi Y, Soto-Quiros ME, Avila L, Lasky-Su J, et al: MMP12, lung function, and COPD in high-risk populations. $N$ Engl J Hum Serv 2009, 361(27):2599-2608. Epub 2009/12/19.

8. Rosas IO, Richards TJ, Konishi K, Zhang Y, Gibson K, Lokshin AE, et al: MMP1 and MMP7 as potential peripheral blood biomarkers in idiopathic pulmonary fibrosis. PLoS Med 2008, 5(4):e93. Epub 2008/05/02.

9. Jones CB, Sane DC, Herrington DM: Matrix metalloproteinases: a review of their structure and role in acute coronary syndrome. Cardiovasc Res 2003, 59(4):812-823. Epub 2003/10/14.

10. Death AK, Nakhla S, McGrath KC, Martell S, Yue DK, Jessup W, et al: Nitroglycerin upregulates matrix metalloproteinase expression by human macrophages. J Am Coll Cardiol 2002, 39(12):1943-1950. Epub 2002/06/27

11. Imai K, Dalal SS, Chen ES, Downey R, Schulman LL, Ginsburg M, et al: Human collagenase (matrix metalloproteinase-1) expression in the lungs of patients with emphysema. Am J Respir Crit Care Med 2001, 163(3 Pt 1):786-791. Epub 2001/03/20.

12. Geraghty $P$, Dabo AJ, D'Armiento J: TLR4 protein contributes to cigarette smoke-induced matrix metalloproteinase-1 (MMP-1) expression in chronic obstructive pulmonary disease. J Biol Chem 2011, 286(34):30211-30218. Epub 2011/07/07.
13. Vandenbroucke RE, Dejonckheere $\mathrm{E}$, Libert $\mathrm{C}$ : A therapeutic role for matrix metalloproteinase inhibitors in lung diseases? Eur Respir J, 38(5):1200-1214. Epub 2011/06/11.

14. Martin MD, Matrisian LM: The other side of MMPs: protective roles in tumor progression. Cancer Metastasis Rev 2007, 26(3-4):717-724. Epub 2007/08/25.

15. Elkington PT, Friedland JS: Matrix metalloproteinases in destructive pulmonary pathology. Thorax 2006, 61(3):259-266. Epub 2005/10/18.

16. Joos L, He JQ, Shepherdson MB, Connett JE, Anthonisen NR, Pare PD, et al: The role of matrix metalloproteinase polymorphisms in the rate of decline in lung function. Hum Mol Genet 2002, 11(5):569-576. Epub 2002/03/05.

17. Gosselink JV, Hayashi S, Elliott WM, Xing L, Chan B, Yang L, et al: Differential expression of tissue repair genes in the pathogenesis of chronic obstructive pulmonary disease. Am J Respir Crit Care Med 2010, 181(12):1329-1335. Epub 2010/01/16

18. Maclay JD, McAllister DA, Rabinovich R, Haq I, Maxwell S, Hartland S, et al: Systemic elastin degradation in chronic obstructive pulmonary disease. Thorax 2012, 67(7):606-612. Epub 2012/03/01.

19. Weiden MD, Ferrier N, Nolan A, Rom WN, Comfort A, Gustave J, et al: Obstructive airways disease with air trapping among firefighters exposed to World Trade Center dust. Chest 2010, 137(3):566-574. Epub 2009/10/13.

20. Weiden MD, Naveed B, Kwon S, Cho SJ, Comfort AL, Prezant DJ, et al: Cardiovascular biomarkers predict susceptibility to lung injury in World Trade Center dust-exposed firefighters. Eur Respir J 2013, 41(5):1023-30. Epub 2012/08/21.

21. Rundle $A G$, Vineis $P$, Ahsan $H$ : Design options for molecular epidemiology research within cohort studies. Cancer Epidemiol Biomarkers Prev 2005, 14(8):1899-1907. Epub 2005/08/17.

22. Shaker SB, von Wachenfeldt KA, Larsson S, Mile I, Persdotter S, Dahlback M, et al: Identification of patients with chronic obstructive pulmonary disease (COPD) by measurement of plasma biomarkers. Clin Respir J 2008, 2(1):17-25. Epub 2008/01/01.

23. Ziora D, Dworniczak S, Kozielski J: Induced sputum metalloproteinases and their inhibitors in relation to exhaled nitrogen oxide and sputum nitric oxides and other inflammatory cytokines in patients with chronic obstructive pulmonary disease. J Physiol Pharmacol 2008, 59(Suppl 6):809-817. Epub 2009/02/28.

24. Engstrom G, Lindberg C, Gerhardssonde Verdier M, Nihlen U, Anderson M, Svartengren $\mathrm{M}$, et al: Blood biomarkers and measures of pulmonary function-a study from the Swedish twin registry. Respir Med 2012, 106(9):1250-1257. Epub 2012/06/13.

25. Ambalavanan N, Nicola T, Li P, Bulger A, Murphy-Ullrich J, Oparil S, et al: Role of matrix metalloproteinase-2 in newborn mouse lungs under hypoxic conditions. Pediatr Res 2008, 63(1):26-32. Epub 2007/11/29.

26. Tchougounova E, Lundequist A, Fajardo I, Winberg JO, Abrink M, Pejler G: A key role for mast cell chymase in the activation of pro-matrix metalloprotease-9 and pro-matrix metalloprotease-2. J Biol Chem 2005, 280(10):9291-9296. Epub 2004/12/24

27. Kheradmand F, Kiss A, Xu J, Lee SH, Kolattukudy PE, Corry DB: A proteaseactivated pathway underlying Th cell type 2 activation and allergic lung disease. J Immunol 2002, 169(10):5904-5911. Epub 2002/11/08.

28. Caplan-Shaw CE, Yee H, Rogers L, Abraham JL, Parsia SS, Naidich DP, et al: Lung pathologic findings in a local residential and working community exposed to World Trade Center dust, gas, and fumes. J Occup Environ Med 2011, 53(9):981-991. Epub 2011/08/24.

29. Corry DB, Rishi K, Kanellis J, Kiss A, Song Lz LZ, Xu J, et al: Decreased allergic lung inflammatory cell egression and increased susceptibility to asphyxiation in MMP2-deficiency. Nat Immunol 2002, 3(4):347-353. Epub 2002/03/12.

30. Gonzalez-Lopez A, Astudillo A, Garcia-Prieto E, Fernandez-Garcia MS, Lopez-Vazquez A, Batalla-Solis E, et al: Inflammation and matrix remodeling during repair of ventilator-induced lung injury. Am J Physiol Lung Cell Mol Physiol 2011, 301(4):L500-L509. Epub 2011/07/12.

31. D'Armiento J, Dalal SS, Okada Y, Berg RA, Chada K: Collagenase expression in the lungs of transgenic mice causes pulmonary emphysema. Cell 1992, 71(6):955-961. Epub 1992/12/11.

doi:10.1186/1465-9921-15-5

Cite this article as: Nolan et al:: MMP-2 and TIMP-1 predict healing of WTC-lung injury in New York City firefighters. Respiratory Research 2014 15:5. 